\title{
Giant basal cell carcinoma of the left lateral neck
}

\author{
Bongsoo Baik ${ }^{1}$, \\ Sulki Park ${ }^{1}$, \\ Soyoung Ji', \\ Sunyoung Kim² \\ Departments of ${ }^{1}$ Plastic and \\ Reconstructive Surgery and ${ }^{2}$ Pathology, \\ Dongkang Medical Center, Ulsan, \\ Korea
}

\begin{abstract}
Basal cell carcinoma is a malignant epithelial neoplasm of the skin and the most common human skin cancer. It is generally associated with a good prognosis. In this case report, a giant basal cell carcinoma of the nodulo-ulcerative type showing wide ulceration with marginal multiple small nodules, is presented. It was trapezoidal in shape, having dimensions of $7 \mathrm{~cm}$ at the greatest basal width, $6 \mathrm{~cm}$ vertically with different anterior and posterior margin dimensions, and $5 \mathrm{~cm}$ horizontally at the top margin. After wide excision of the lesion including 5-10 mm safety margins, the wound was reconstructed with a local skin flap and split-thickness skin graft. The reconstructed wound healed well without recurrence for 1 year.
\end{abstract}

Abbreviations: BCC, basal cell carcinoma; GBCC, giant basal cell carcinoma; SCM, sternocleidomastoid.

Keywords: Basal cell carcinoma / Head and neck neoplasm / Skin neoplasms

\section{INTRODUCTION}

Basal cell carcinoma (BCC) and squamous cell carcinoma account for $77 \%$ and $20 \%$, respectively, of all skin cancers, with melanoma and rare tumors constituting the remaining 3\% [1]. Among the rare tumors it has been reported that the incidence of giant basal cell carcinoma (GBCC) was $0.5 \%$ of all basal cell cancers [2]. GBCC is defined as a lesion greater than $5 \mathrm{~cm}$ in its longest dimension through the center, independent of histological subtype, local invasion, or metastasis [3]. The American Joint Cancer Committee classifies GBCCs as T3 tumors [4,5]. Typically, BCCs are characterized by slow growth, minimal local invasiveness, lower morbidity, and a high cure rate. In contrast, GBCC is a rare and aggressive form of BCC, which usually carries a higher risk of complications and mortality $[6,7]$.

Further in contrast to BCCs, GBCCs often occur on skin that is less exposed to sunlight, such as the back, shoulders, legs, and thighs [8]. The growing tumor is often accompanied with ulcer-

Correspondence: Bongsoo Baik

Department of Plastic and Reconstructive Surgery, Dongkang Medical Center,

239 Taehwa-ro, Jung-gu, Ulsan 44455, Korea

E-mail: bsbaiksy@naver.com

Received May 8, 2021 / Revised May 29, 2021 / Accepted June 20, 2021 ation and necrosis [9] and GBCC of the head and neck in particular, is often accompanied with ulceration and bad odor [2].

Here, we report a case of GBCC involving the left lateral neck and mandibular border.

\section{CASE REPORT}

A 74-year-old man visited the plastic surgery department with a wide nodulo-ulcerative lesion on the left lateral neck and mandibular border. The patient had been presented a small palpating mass on his left upper neck 14 years ago. However, he did not undergo surgery at the time for socioeconomic reason. Over the past 3 years, the mass grew rapidly with central ulceration and minor discharge from the ulcerated wound.

Physical examination revealed a wide ulcerated lesion covered with an unwashed, infected pseudomembrane. Multiple small nodules were found along the margin. The inferior margin of the lesion was located on the left lateral mid-neck and was $7 \mathrm{~cm}$ in its greatest dimension. The posterior vertical margin was about $6 \mathrm{~cm}$ and located $1.5-2.5 \mathrm{~cm}$ anteriorly to the lateral hairline of the occipital scalp. The anterior vertical margin was located at the lateral cheek along the posterior mandibular border 
and anterior neck. It was difficult to measure its length because of the zig-zag shape involving the skin. The superior margin of the lesion involved the lowest part of the earlobe and the retroauricular area and was $5 \mathrm{~cm}$ in dimension. The central ulcerated wound was partly adherent with the underlying soft tissue.

A punch biopsy of the nodule showed BCC. A computed tomography scan of the neck showed a subcutaneous mass measuring $5 \mathrm{~cm}$ in diameter in the anterio-posterior plane with central skin ulceration. The lesion was suspected of having invaded the sternocleidomastoid (SCM) muscle and the inferior pole of the parotid gland (Fig. 1).

The incision was marked with a safety margin of $5 \mathrm{~mm}$ from the visible tumor on the inferior, posterior, and superior portions of the lesion, and a $10 \mathrm{~mm}$ safety margin on the mandibular margin and anterior neck (Fig. 2). The anterior incision was

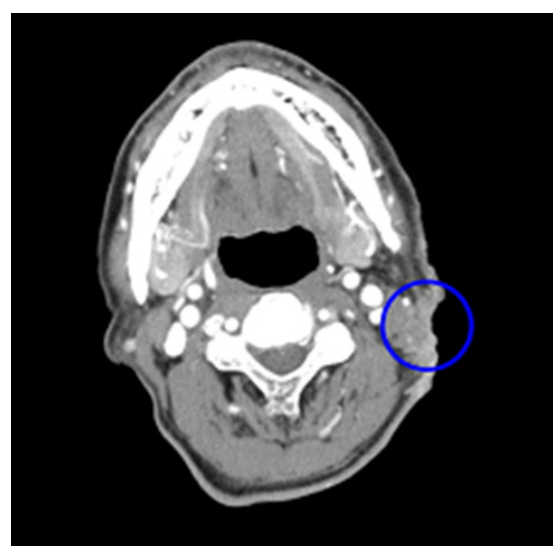

Fig. 1. Preoperative enhanced computed tomography finding. A 5 $\mathrm{cm}$ mass on the left lateral neck, and skin thickening with central ulceration is seen (blue circle).

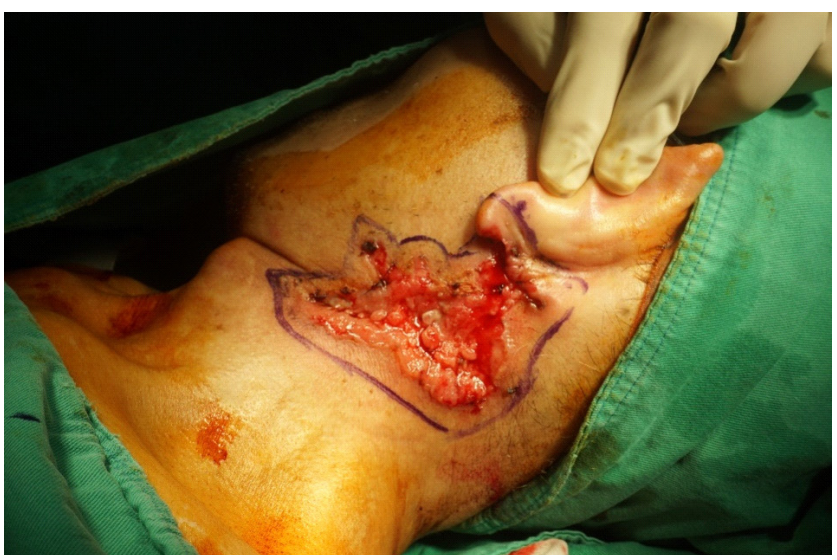

Fig. 2. Preoperative photograph. The preoperative ulcerated wound was covered with an infected pseudomembrane and multiple small nodules. The incision design was marked with a safety margin of 5 $\mathrm{mm}$ from the visible tumor on the inferior, posterior, and superior portions of the lesion, and a safety margin of $10 \mathrm{~mm}$ on the mandibular margin and anterior neck. deep and included the superficial musculoaponeurotic system on the posterior portion of the mandibular ramus and the platysma muscle on the anterior neck. Other incisions were made deep into the fascia. When the tissue including the tumor was elevated from the underlying tissue, the tumor was found to have invaded the superficial lobe of the parotid gland. Consequently, partial parotidectomy was performed without injuring
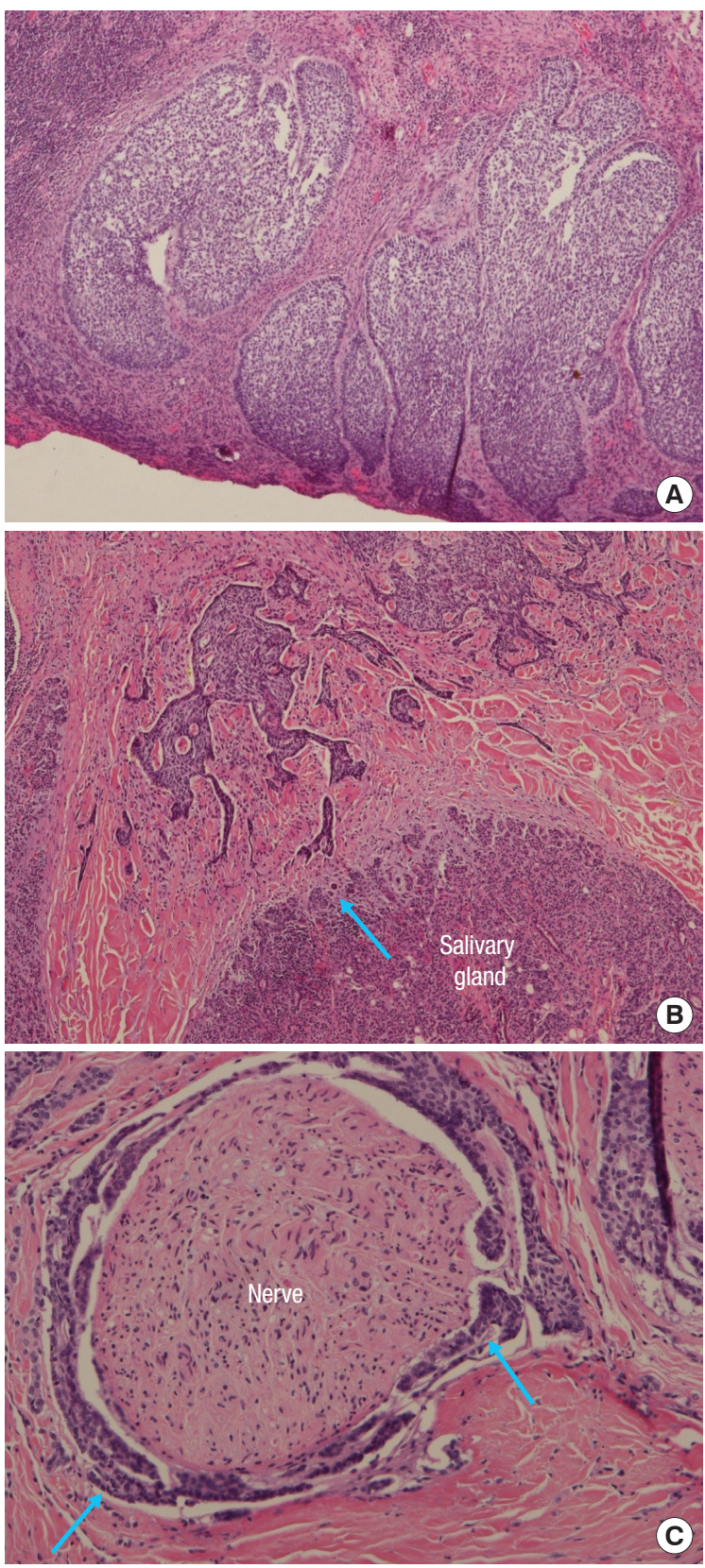

Fig. 3. Histological findings. (A) Photomicrograph shows nodular basal cell carcinoma with peripheral palisading and ulceration (H\&E, $\times 40$ ), (B) focal infiltration to the salivary gland (blue arrow) $(\mathrm{H} \& \mathrm{E}, \times 40)$, and $(\mathrm{C})$ perineurial tumor infiltration (blue arrows) of a nerve $(\mathrm{H} \& \mathrm{E}, \times 100)$. 

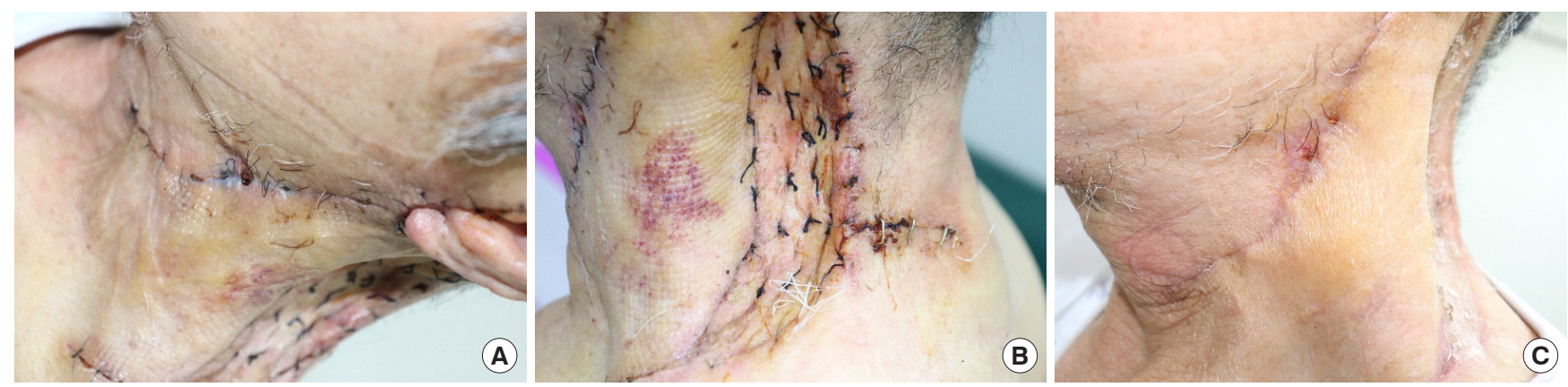

Fig. 4. Postoperative photographs. Seven days after flap transfer and skin graft: (A) the flap survived well and (B) the skin graft was well taken. (C) At postoperative day 21, the wounds were healed and the skin flap covered the mandibular margin and the lateral neck except for the narrow area showing the vertical skin graft.

the facial nerve. The tumor had also invaded the fascia of the SCM muscle and therefore the invaded fascia and some part of the SCM muscle were excised.

The excised specimens were sent to the Pathology department for frozen sectioning. The frozen diagnosis was "Focally suspicious positive basal resection margin." The final pathologic findings showed BCC, nodular pattern mixed with occasionally micronodular and infiltrating patterns (Fig. $3 \mathrm{~A}$ ), $6.8 \times 6.7 \mathrm{~cm}$ in dimension, invading to the subcutaneous tissue, skeletal muscle and focal salivary gland (Fig. 3B), $1.3 \mathrm{~cm}$ in depth. The tumor showed perineural invasion (Fig. 3C), but no lymphovascular invasion. No metastasis on the regional lymph nodes is noted. The resection margins revealed focally tumor involvement on the basal margin, but clear side resection margins.

After further excision of the affected soft tissue, a horizontal local skin flap with a $7-\mathrm{cm}$ pedicle base and $15-\mathrm{cm}$ flap length was elevated to include the skin of the lower posterior portion of the wound. The skin flap was transferred to cover the lateral cheek, the anterolateral neck including the SCM muscle, and the retroauricular area. The remaining open wound was covered with a split-thickness skin graft. The transferred flap and the skin graft healed well without complications (Fig. 4).

\section{DISCUSSION}

The four goals of carcinoma treatment include complete tumor excision, prevention of recurrence, preservation of function, and optimal cosmesis. The TNM classification based on the American Joint Committee on Cancer, has been widely used to plan treatment and estimate patient prognosis. According to the modified 8th edition, $\mathrm{T}$ categories are based on independent risk factors for poor prognosis [10]. The present case was at the T3 stage with deep invasion ( $>4 \mathrm{~cm}$ in diameter and invasion beyond subcutaneous fat).

Treatment of GBCC is not significantly different from that of
BCC. Surgical excision is a basic and important approach with an average success rate of $90 \%$ to $91 \%$ for skin cancer [11]. Although BCC treatment is relatively simple and has a very low mortality rate [12], GBCC requires extensive resection and reconstruction. However, the deep and invasive nature of GBCC accompanied by rapid growth with a high risk of metastasis, makes it difficult to manage. In particular, GBCC involving the head and neck region, with its many important stuctures including nerves, glands, and muscle, is a surgical challenge.

This patient's GBCC was associated with a risk of incomplete excision due to its large size and widespread invasion of extradermal structures. In addition, removal of tumors of this sort can lead to large soft tissue defects, which can render reconstruction difficult. Accordingly, our goal was to eliminate the risk of functional challenges and cosmetic concerns after surgery.

Despite the potential for incomplete resection and difficult wound reconstruction, the tumor was removed with the invaded fascia, as well as some part of the SCM, and a portion of the salivary gland. However, pathology results showed focal invasion to the skeletal muscle and superficial parotid gland as well. After further resection of the SCM muscle and superficial lobe of the parotid gland, a long and wide local flap was transferred to the lateral cheek and post-auricular area. The insufficiently covered wound on the posterior neck was further treated to obtain complete coverage with split-thickness skin graft. The wounds healed successfully without infection, hematoma, facial nerve palsy, or flap necrosis.

Unlike BCCs, GBCCs are more likely to have an aggressive histologic subtype (morpheaform, micronodular), and therefore complete resections are challenging.

Reconstruction options and postoperative cosmetic aspects must also be considered. Nevertheless, it is important to remove the tumor completely and reduce the risk of recurrence. The appropriate reconstruction method should be selected based on 
primary wound healing, protection of critical structures, contour restoration, and functional and aesthetic outcomes. A number of reconstruction methods are available to deal with large and complex defects. Local flaps and/or skin grafts are straightforward and effective procedures. When a local flap is not feasible, use of a free flap with microvascular surgery will be next choice.

\section{NOTES}

\section{Conflict of interest}

No potential conflict of interest relevant to this article was reported.

\section{Ethical approval}

The study was approved by the Institutional Review Board of Dongkang Medical Center (IRB No. 2021-03-01) and performed in accordance with the principles of the Declaration of Helsinki. Written informed consent was obtained.

\section{Patient consent}

The patient provided written informed consent for the publication and the use of his images.

\section{ORCID}

\section{Bongsoo Baik}

Sulki Park

Soyoung Ji

Sunyoung Kim

\section{Author contribution}

Conceptualization: BB. Resources: SK. Project administration: SP. Visualization: SP. Writing - original draft: SP. Writing - review \& editing: BS, SJ.

\section{REFERENCES}

1. Park YJ, Kwon GH, Kim JO, Kim NK, Ryu WS, Lee KS. A ret- rospective study of changes in skin cancer characteristics over 11 years. Arch Craniofac Surg 2020;21:87-91.

2. Arslan H, Guzel MZ, Cnar C. Treatment of giant basal cell carcinomas of the head and neck with aggressive resection and complex reconstruction. J Craniofac Surg 2012;23:1634-7.

3. Mott SE, Hunter WJ, Silva E, Huerter CJ. Approach to management of giant basal cell carcinomas. Cutis 2017;99:356-62.

4. Manstein CH, Gottlieb N, Manstein ME, Manstein G. Giant basal cell carcinoma: a series of seven T3 tumors without metastasis. Plast Reconstr Surg 2000;106:653-6.

5. Amin MB, Greene FL, Edge SB, Compton CC, Gershenwald JE, Brookland RK, et al. The eighth edition AJCC Cancer Staging Manual: continuing to build a bridge from a populationbased to a more "personalized" approach to cancer staging. CA Cancer J Clin 2017;67:93-9.

6. Maimaiti A, Mijiti A, Yarbag A, Moming A. Giant basal cell carcinoma of the face: surgical management and challenges for reconstruction. J Laryngol Otol 2016;130:176-82.

7. Archontaki M, Stavrianos SD, Korkolis DP, Arnogiannaki N, Vassiliadis V, Liapakis IE, et al. Giant basal cell carcinoma: clinicopathological analysis of 51 cases and review of the literature. Anticancer Res 2009;29:2655-63.

8. Heo YS, Yoon JH, Choi JE, Ahn HH, Kye YC, Seo SH. A case of superficial giant basal cell carcinoma with satellite lesions on scalp. Ann Dermatol 2011;23 Suppl 1:S111-5.

9. Wee SJ, Park MC, Chung CM. Basal cell carcinoma misdiagnosed as trichoepithelioma. Arch Craniofac Surg 2020;21:2025.

10. Skulsky SL, O’Sullivan B, McArdle O, Leader M, Roche M, Conlon PJ, et al. Review of high-risk features of cutaneous squamous cell carcinoma and discrepancies between the American Joint Committee on Cancer and NCCN clinical practice guidelines in oncology. Head Neck 2017;39:578-94.

11. Wolf DJ, Zitelli JA. Surgical margins for basal cell carcinoma. Arch Dermatol 1987;123:340-4.

12. Kiiski V, de Vries E, Flohil SC, Bijl MJ, Hofman A, Stricker BH, et al. Risk factors for single and multiple basal cell carcinomas. Arch Dermatol 2010;146:848-55. 